\title{
COMPLETENESS AND BASIS PROPERTIES OF COMPLEX EXPONENTIALS
}

\author{
BY
}

RAYMOND M. REDHEFFER AND ROBERT M. YOUNG

\begin{abstract}
This paper is concerned with what might be termed the "fine structure" of the completeness and basis properties of complex exponentials. We give new criteria for two sequences to have the same excess in the sense of Paley and Wiener, a result that illuminates and supplements a well-known completeness criterion of Levinson, and new examples and counterexamples pertaining to Riesz bases.
\end{abstract}

1. Introduction. There is an extensive literature on the completeness and expansion properties of sets of complex exponentials over a finite interval of the real axis (see, e.g., [5 and 7] and the references therein). The present work continues this investigation.

A system $\left\{e^{i \lambda_{n} t}\right\}$ of complex exponentials is said to be complete $L^{p}(-a, a)$, where $1 \leqslant p \leqslant \infty$, if the relations

$$
\int_{-a}^{a} f(t) e^{i \lambda_{n} t} d t=0 \quad(n=1,2,3, \ldots),
$$

with $f \in L^{p}$, imply that $f=0$ a.e. The system is closed $L^{p}(-a, a)$ if every $f \in L^{p}$ on this interval can be approximated in $L^{p}$ norm by linear combinations of the functions $e^{i \lambda_{n} t}$. By duality, if $1<p<\infty$ then closure $L^{p}$ is equivalent to completeness $L^{q}$, where $q$ is the conjugate exponent, i.e., $1 / p+1 / q=1$. The slight lack of symmetry for $p=1$ or $p=\infty$ is overwhelmed by the effect of adding or removing a single $\lambda$.

If the system $\left\{e^{i \lambda_{n} t}\right\}$ is complete $L^{p}(-a, a)$ but fails to be complete on the removal of a single term, then it will be called exact. If it becomes exact when $E$ terms are removed, then we say it has excess $E$ or $E(\lambda)$. The deficiency is defined similarly, or as a "negative excess." By convention, $E=\infty$ if arbitrarily many terms can be removed without losing completeness, and $E=-\infty$ if arbitrarily many terms can be adjoined without getting completeness. Observe that the quantity $E$ depends on both $p$ and $a$; although this dependence is not built into the notation, the context will make its use clear.

In the first part of this paper (§§2-5) we obtain estimates for $|E(\lambda)-E(\mu)|$ in terms of $\left|\lambda_{n}-\mu_{n}\right|$. By convention, an inequality of the form $|E(\lambda)-E(\mu)| \leqslant A$ shall mean: Either $E(\lambda)$ and $E(\mu)$ are both $\infty$, or they are both $-\infty$, or they are both finite and satisfy the stated inequality. Some of the results appeared without

Received by the editors November 16, 1981.

1980 Mathematics Subject Classification. Primary 42A64; Secondary 42 A60.

Key words and phrases. Complete system, excess, exact system, biorthogonal, Riesz basis. 
proof in [5]; others are extensions and refinements of work done by one of the authors and others in $[1,3,4$ and 5]. In the second part of the paper (\$\$6-9) we investigate the basis properties of systems of complex exponentials that are in some sense "close to" the trigonometric system $\left\{e^{i n t}\right\}$.

In $§ 2$, we consider the mapping

$$
T: f \rightarrow e^{-i \lambda x} \int_{-a}^{x} e^{i \lambda t} f(t) d t .
$$

Here, $\lambda$ is a fixed complex number and $f$ belongs to the subspace of $L^{p}(-a, a)$, $1 \leqslant p \leqslant \infty$, for which $\int_{-a}^{a} e^{i \lambda t} f(t) d t=0$. Then $T$ is a bounded linear operator into $L^{p}(-a, a)$. While the exact determination of $\|T\|$ is an open problem, we show that when $p=2$,

$$
\|T\|=2 a /\left(\pi^{2}+4|\operatorname{Im} \lambda|^{2} a^{2}\right)^{1 / 2},
$$

and that, in general, $\|T\| \leqslant \min \left(2 a,|\operatorname{Im} \lambda|^{-1}\right)$.

The results of $\S 2$ are used in $\S 3$ to compare the excesses of two sequences $\left\{e^{i \lambda_{n} t}\right\}$ and $\left\{e^{i \mu_{n} t}\right\}$. It is shown, for example, that if the points $\lambda_{n}$ and $\mu_{n}$ lie in the half-plane $\operatorname{Im} z \geqslant c$ and if

$$
\limsup _{n \rightarrow \infty} \frac{\left|\lambda_{1}-\mu_{1}\right|+\cdots+\left|\lambda_{n}-\mu_{n}\right|}{\log n}<\infty,
$$

then the $L^{p}$ excesses of the two sequences satisfy $|E(\lambda)-E(\mu)| \leqslant 1$.

For a real increasing sequence $\left\{\lambda_{n}\right\}$ of nonzero numbers, the counting function $\Lambda(u)$ is the number of $\lambda_{k}$ on the interval $(0, u]$, counted negatively for negative $u$. An important use of the counting function is in the formula

$$
\log |F(x)|=\int_{-\infty}^{\infty} \frac{\Lambda(u)}{u} \frac{x}{x-u} d u,
$$

where

$$
F(x)=\lim _{R \rightarrow \infty} \prod_{\left|\lambda_{n}\right|<R}\left(1-\frac{x}{\lambda_{n}}\right) \text { and } \Lambda(u)=o\left(u^{2}\right) .
$$

In $\S 4$, we investigate this integral under the assumption that $\Lambda(u)=[u-\phi(u)]$ where $\phi: R \rightarrow R$ is a twice differentiable, bounded function, $\phi^{\prime}(u)<1,0<\phi(0)<1$ (this ensures that $u \Lambda(u) \geqslant 0$ and that no $\lambda_{n}$ is zero), and $\sum_{-\infty}^{\infty}\left|\phi^{\prime \prime}\left(\xi_{n}\right)\right|<\infty$ whenever $\xi_{n} \in\left(\lambda_{n}, \lambda_{n+1}\right)$. In many applications, it is desirable to replace $\Lambda(u)$ by the simpler expression $u-\phi(u)-\frac{1}{2}$. In this case, the error $E(x)$ introduced in the integral is shown to satisfy $|E(x)| \leqslant O(1)$ whenever $\inf _{n}\left|x-\lambda_{n}\right| \geqslant \varepsilon$, and $E(x) \leqslant$ $O(1)$ for all $x$.

A classical result of Levinson [3,p. 6] states that the system $\left\{e^{i \lambda_{n} t}\right\}$ is complete $L^{p}(-\pi, \pi)$ if $\left|\lambda_{n}\right| \leqslant|n|+1 / 2 q$, and that the constant $1 / 2 q$ cannot be replaced by any larger number. It is not difficult to show that completeness continues to hold if

$$
\left|\lambda_{n}\right| \leqslant|n|+\frac{1}{2 q}+\phi(|n|)
$$


where $\phi$ is a sufficiently regular function for which $\Sigma_{1}^{\infty} \phi(n) / n<\infty$. In $\S 5$, using the results of $\S 4$, we investigate the case $\phi(n)=\beta / \log n, \beta \neq 0$, which just fails to satisfy this criterion.

For the remainder of the paper, we restrict attention to the basis properties of a system $\left\{e^{i \lambda_{n} t}\right\}_{-\infty}^{\infty}$ of complex exponentials in $L^{2}(-\pi, \pi)$. Such a system is said to be a Riesz basis for $L^{2}(-\pi, \pi)$ if the mapping $e^{i n t} \rightarrow e^{i \lambda_{n} t}(-\infty<n<\infty)$ can be extended to an isomorphism on all of $L^{2}(-\pi, \pi)$. If this is the case, then each function $f$ in $L^{2}(-\pi, \pi)$ will have a unique nonharmonic Fourier expansion

$$
f(t)=\sum_{-\infty}^{\infty} c_{n} e^{i \lambda_{n} t} \quad \text { (in the mean) }
$$

with $\left\{c_{n}\right\} \in l^{2}$.

The possibility of such expansions was discovered by Paley and Wiener, who showed that the system $\left\{e^{i \lambda_{n} t}\right\}$ is a Riesz basis for $L^{2}(-\pi, \pi)$ whenever each $\lambda_{n}$ is real and $\left|\lambda_{n}-n\right| \leqslant L<1 / \pi^{2}$. Ultimately, Kadec showed that the constant $1 / \pi^{2}$ could be replaced by $\frac{1}{4}$. That $\frac{1}{4}$ is in fact the "best possible" constant follows from the fact that the system $\left\{e^{ \pm i(n-1 / 4) t}\right\}_{n=1}^{\infty}$ is already exact in $L^{2}(-\pi, \pi)$. (For a comprehensive introduction to the theory of nonharmonic Fourier series, including proofs of these assertions, see [7].)

Riesz bases constitute the largest and most tractable class of bases known. In fact, there is no known example of a basis of complex exponentials for $L^{2}(-\pi, \pi)$ which is not a Riesz basis.

In §7, we investigate some of the ways in which Kadec's result is the best possible. In particular, we show that the condition $\left|\lambda_{n}-n\right|<\frac{1}{4}(-\infty<n<\infty)$ is not strong enough to ensure that the system $\left\{e^{i \lambda_{n} t}\right\}$ is a Riesz basis for $L^{2}(-\pi, \pi)$, even if it is exact. For this purpose, we analyze in detail, in $\$ 6$, canonical products of the form

$$
F(z)=z \prod_{n=1}^{\infty}\left(1-\frac{z^{2}}{(n+\varepsilon)^{2}}\right), \quad \varepsilon>0,
$$

obtaining both an integral representation for $F(z)$ and a simple formula for $F^{\prime}(n+\varepsilon)$.

If

$$
\lambda_{n}= \begin{cases}n+\frac{1}{4}, & n>0, \\ 0, & n=0, \\ n-\frac{1}{4}, & n<0,\end{cases}
$$

then the system $\left\{e^{i \lambda_{n} t}\right\}$ is a reasonable candidate for a "non-Riesz" basis of complex exponentials. We investigate this assertion in $\S 8$, showing in particular that the system possesses a bounded biorthogonal system. This is then related to the solution of certain interpolation problems in a classical Hilbert space of entire functions.

An entire function $F(z)$ of exponential type $\pi$ is said to be of sine type if (1) its zeros $\lambda_{n}$ are separated, i.e., $\inf _{n \neq m}\left|\lambda_{n}-\lambda_{m}\right|>0$, and (2) there exist positive constants $A, B$ and $H$ such that

$$
A e^{\pi|y|} \leqslant|F(x+i y)| \leqslant B e^{\pi|y|}
$$


whenever $x$ and $y$ are real and $|y| \geqslant H$. It is well known that if $\left\{\lambda_{n}\right\}$ is the set of zeros of a function of sine type, then the system $\left\{e^{i \lambda_{n} t}\right\}$ is a Riesz basis for $L^{2}(-\pi, \pi)$. In $\S 9$ we exhibit a large class of Riesz bases of complex exponentials that cannot be obtained in this way.

2. An integral inequality. With $a>0$ and $1 \leqslant p \leqslant \infty$ let $f \in L^{p}(-a, a)$ and let

$$
y(x)=e^{-i \lambda x} \int_{-a}^{x} e^{i \lambda t} f(t) d t, \quad y(a)=0 .
$$

where $\lambda=\rho+i \sigma$ is a given complex number. The question addressed here is the following: What is the best constant $K=K(p, a, \lambda)$ such that $\|y\|_{p} \leqslant K\|f\|_{p}$ where $\|\cdot\|_{p}$ denotes the $L^{p}$ norm on $(-a, a)$ ? This question underlies the results stated without proof at the end of [4], some of those in [1] and their refinements in [5].

Let us begin by establishing the property

$$
K(p, a, \lambda)=a K(p, 1,|\sigma| a) .
$$

If the inequality

$$
\int_{-a}^{a} e^{p \sigma x}\left|\int_{-a}^{x} e^{-\sigma t} e^{i \rho t} f(t) d t\right|^{p} d x \leqslant K^{p} \int_{-a}^{a}\left|e^{i \rho t} f(t)\right|^{p} d t
$$

is to hold for all $f \in L^{p}$ such that

$$
\int_{-a}^{a} e^{-\sigma t} e^{i \rho t} f(t) d t=0
$$

this is equivalent to a condition of the same kind applied to the function $g(t)=$ $f(t) e^{i \rho t}$. Since $|g|=|f|$ we conclude that $\rho=0$ involves no loss of generality, or in other words that $K(p, a, \lambda)=K(p, a, \sigma)$. To see that the result depends only on $|\sigma|$ let $\rho=i \sigma$ and use the fact that $y(a)=0$. By setting $t=-s$ in the inner integral it is found that the inequality with $\sigma$ and $f(t)$ is equivalent to the inequality with $-\sigma$ and $f(-t)$, including the same side condition $y(a)=0$. Making the change of variable $t=c s$, where $c$ is a suitable constant, we get (1).

Let us then take $\lambda=i \sigma$ with $\sigma \geqslant 0$. If $y$ rather than $f$ is viewed as the unknown function the inequality is equivalent to

$$
\int_{-a}^{a}|y|^{p} d x \leqslant K^{p} \int_{-a}^{a}\left|y^{\prime}-\sigma y\right|^{p} d x, \quad y(-a)=y(a)=0 .
$$

This formulation together with (1) leads to the value

$$
K(2, a, \lambda)=\frac{2 a}{\left(\pi^{2}+4 \sigma^{2} a^{2}\right)^{1 / 2}}
$$

which was given without proof in [5]. To see this, let $p=2$ in (2) and write $y=u+i v$. It is found that the inequality holds for $y$ if and only if it holds for $u$ and $v$. Hence, without loss of generality, $y$ is real. In that case the term $-2 \sigma y y^{\prime}$ in the expansion of the term on the right integrates to 0 and the inequality reduces to

$$
\left(1-K^{2} \sigma^{2}\right) \int_{-a}^{a} y^{2} d x \leqslant K^{2} \int_{-a}^{a}\left(y^{\prime}\right)^{2} d x .
$$


Theorem 257 of [2] gives $K^{2}=1-K^{2} \sigma^{2}$ when $a=\pi / 2$, and the result for arbitrary $a$ is now given by (1).

When $\sigma=0$ the reformulation (2) again falls into a standard pattern. Applying [2, Theorem 256] on $(-a, 0)$ and on $(0, a)$ and adding, we get the value

$$
K(p, a, 0)=\frac{p}{(p-1)^{1 / p}} \frac{a}{\pi} \sin \frac{\pi}{p}, \quad p=\text { even integer }
$$

for $a=2$. The result for general $a$ follows from (1). That the constant is sharp follows from the fact that Theorem 256 is sharp.

The determination of $K(p, a, \lambda)$ for $\sigma>0$ and arbitrary $p$ is left as an open problem. However, we shall establish the inequality

$$
K(p, a, \lambda) \leqslant \min \left(2 a,|\sigma|^{-1}\right)
$$

which was stated without proof in [5]. A routine use of the Hölder inequality gives

$$
K(p, a, \lambda) \leqslant \min \left(2 a,(2 a)^{1 / p}(q \sigma)^{-1 / q}\right)
$$

and even a somewhat stronger result [1], but (4) is more difficult.

The proof is as follows. Let $\lambda=i \sigma$ where $\sigma>0$ is fixed and define a transformation $T$ by

$$
T f=e^{-\sigma x} \int_{-a}^{x} e^{\sigma t} f(t) d t
$$

Then

$$
\|T f\|_{\infty} \leqslant\|f\|_{\infty} \cdot \sup _{x} e^{-\sigma x} \int_{-a}^{x} e^{\sigma t} d t \leqslant \min \left(2 a, \sigma^{-1}\right)\|f\|_{\infty},
$$

where the second inequality follows from $1-e^{-s}<\min (1, s)$ for $s>0$. Furthermore

$$
\|T f\|_{1} \leqslant \int_{-a}^{a} e^{-\sigma x} \int_{-a}^{x} e^{\sigma t}|f(t)| d t d x \leqslant \min \left(2 a, \sigma^{-1}\right)\|f\|_{1}
$$

where the second inequality follows from Fubini's theorem together with the same inequality for $1-e^{-s}$ as was used above. This gives the desired result for $p=1$ and $p=\infty$. Now comes an important point. The only use of the side condition $y(a)=0$ was to permit the assumption that $\sigma>0$. Apart from this, the condition $y(a)=0$ plays no role in the above estimates for the norm of the linear operator $T$. Accordingly, we can use the Riesz-Thorin convexity theorem [9] or the Marcinkiewicz interpolation theorem to get the same estimate, with the same constant, for arbitrary $p$. This gives (4). It would be desirable to have a direct proof, without interpolation, but such a proof is not available.

3. Comparison of two sequences. The foregoing discussion has applications to problems of completeness. To illustrate the nature of these we state the following theorem, which is implicit in [5] but has not been formulated hitherto. 
THEOREM 1. Let $\left\{\lambda_{n}\right\}$ and $\left\{\mu_{n}\right\}$ be sequences of complex numbers lying in a half-plane $\operatorname{Im} z \geqslant$ const and so numbered that $\left|\lambda_{n}\right|$ or $\left|\mu_{n}\right|$ increases with $n$. Suppose that

$$
\limsup _{n \rightarrow \infty} \frac{\left|\lambda_{1}-\mu_{1}\right|+\left|\lambda_{2}-\mu_{2}\right|+\cdots+\left|\lambda_{n}-\mu_{n}\right|}{\log n}<\infty .
$$

Then the $L^{p}$ excesses satisfy $|E(\lambda)-E(\mu)| \leqslant 1$.

PROof. Replace $\left\{\lambda_{n}\right\}$ and $\left\{\mu_{n}\right\}$ by $\left\{\lambda_{n}+i b\right\}$ and $\left\{\mu_{n}+i b\right\}$ where $b$ is a positive constant. This alteration does not change the completeness properties of either set and enables us to assume $\operatorname{Im} \lambda_{n}>c, \operatorname{Im} \mu_{n}>c$ where $c$ is as large as may be desired. Let $c \varepsilon_{n}=\left|\mu_{n}-\lambda_{n}\right|$. Then the conclusion $|E(\lambda)-E(\mu)| \leqslant 1$ follows from $\mid \lambda_{n}-$ $\mu_{n} \mid=o(n)$ and

$$
\sum_{n=1}^{\infty} \frac{\left(1+\varepsilon_{1}\right)\left(1+\varepsilon_{2}\right) \cdots\left(1+\varepsilon_{n}\right)}{n^{2}}<\infty .
$$

To see this, let [5, Theorem 19] be stated for $L^{p}$ rather than $L^{2}$, using (4) rather than (3); the proof is unchanged [1]. The result shows that (5) gives $E(\lambda) \leqslant E(\mu)+1$, and by symmetry also $E(\mu) \leqslant E(\lambda)+1$. Since $1+\varepsilon<e^{\varepsilon}$, the hypothesis of Theorem 1 implies (5) when $c$ is large enough, and this completes the proof.

When the $\lambda_{n}$ and $\mu_{n}$ are not required to lie in a half-plane, [5, Theorem 20] implies $|E(\lambda)-E(\mu)|<\infty$ under the remaining hypotheses of Theorem 1. However, the following stronger result is also valid.

THEOREM 2. Let $\left\{\lambda_{n}\right\}$ and $\left\{\mu_{n}\right\}$ be sequences of complex numbers such that $\left|\lambda_{n}\right|$ or $\left|\mu_{n}\right|$ increases with $n$ and

$$
\sum_{j=1}^{n}\left|\operatorname{Re} \lambda_{j}-\operatorname{Re} \mu_{j}\right|=O(\log n), \quad\left|\operatorname{Im} \lambda_{n}-\operatorname{Im} \mu_{n}\right|=O(1) .
$$

Then the $L^{2}$ excesses satisfy $|E(\lambda)-E(\mu)| \leqslant 1$, and hence the $L^{p}$ excesses satisfy $|E(\lambda)-E(\mu)| \leqslant 2$.

Proof. The statement for $L^{p}$ follows from that for $L^{2}$, since $E(\lambda)$ and $E(\mu)$ are monotonic functions of $p$ which change by at most 1 when $p$ ranges from 1 to $\infty$. To prove the result for $p=2$, let us first change $\mu_{n}$ so that $\operatorname{Im} \mu_{n}=\operatorname{Im} \lambda_{n}$ for each $n$, as can be done by the theorem of Elsner and Peterson [5, Theorem 17]. Next fix attention on those $\lambda_{n}$ for which $\left|\operatorname{Im} \lambda_{n}\right| \leqslant c$, where $c$ is a given constant. To these and to the corresponding $\mu_{n}$ we add a term $2 i c$, as can be done by a second use of [5, Theorem 17]. We now have $\left|\operatorname{Im} \lambda_{n}\right| \geqslant c$ and $\left|\operatorname{Im} \mu_{n}\right| \geqslant c$ for all $n$, and the conclusion follows as in the proof of Theorem 1 .

If the hypothesis is strengthened to

$$
\left|\operatorname{Re} \lambda_{n}-\operatorname{Re} \mu_{n}\right|=O(1 / n), \quad\left|\operatorname{Im} \lambda_{n}-\operatorname{Im} \mu_{n}\right|=O(1),
$$

the sum can still have the order $\log n$ allowed in Theorem 2, but the conclusion is $E(\lambda)=E(\mu)$ and $|E(\lambda)-E(\mu)| \leqslant 1$ in the two cases $p=2, p \neq 2$ respectively. This follows from another theorem of Peterson [5, Theorem 18]. The interest of Theorem 2 is that it does not require $\left|\lambda_{n}-\mu_{n}\right|=o(1)$; in fact, $\left|\lambda_{n}-\mu_{n}\right|$ can be, from time to time, almost as large as $\log n$. 
If $\left\{\lambda_{n}\right\}$ and $\left\{\mu_{n}\right\}$ are real, [5, Theorem 65] gives $|E(\lambda)-E(\mu)| \leqslant 1$ under the hypothesis that $p=2$,

$$
\left|\lambda_{n}-\mu_{n}\right|=O\left(\frac{1}{\log n}\right), \quad \sum_{1}^{\infty} \frac{\left|\lambda_{n}-\mu_{n}\right|}{n}<\infty .
$$

The second of these conditions is implied by the hypothesis of Theorem 2 . Whether Theorem 65 can be extended to complex sequences is left as an open problem.

4. The counting function. For any real sequence $\left\{\lambda_{n}\right\}$ the signed counting function $\Lambda(u)$ is the number of $\lambda_{k}$ on the interval $(0, u]$ when $u>0$, and $-\Lambda(u)$ is the number of $\lambda_{k}$ on $[u, 0)$ when $u<0$. Since a finite number of $\lambda_{k}$ can be altered without changing the completeness properties, the restriction $\lambda_{k} \neq 0$ needed for use of this definition does no harm. (For the same reason the following estimates are needed only when $\left|\lambda_{n}\right|,\left|\mu_{n}\right|$ and $|u|$ are all large, provided the early $\lambda$ 's and $\mu$ 's are in 1:1 correspondence. This remark is tacitly used in \$5.)

The deviation of $\left\{\lambda_{n}\right\}$ from $\{n\}$ can be described by $\Lambda(u)-u$ or by $\lambda_{n}-n$. We shall establish a result which allows an easy passage from one of these measures to the other. Let $\phi: R \rightarrow R$ be bounded, let $0<\phi(0)<1$, let $\phi^{\prime}(t)<1$ for $t \in R$, and suppose

$$
\left|\xi_{n}-n\right|<1 \Rightarrow \sum_{-\infty}^{\infty}\left|\phi^{\prime}\left(\xi_{n}\right)\right|<\infty .
$$

Then, as we shall show, the set $\left\{\lambda_{n}\right\}$ with counting function $\Lambda(u)=[u-\phi(u)]$ has the same $L^{p}$ excess as has the set $\left\{\mu_{n}\right\}$ with $\mu_{n}=n+\phi(n)$. For proof, note that $\lambda_{n}$ can be so labeled that $n=\lambda_{n}-\phi\left(\lambda_{n}\right),-\infty<n<\infty$; hence

$$
\lambda_{n}-\mu_{n}=\phi\left(\lambda_{n}\right)-\phi(n)=\left(\lambda_{n}-n\right) \phi^{\prime}\left(\xi_{n}\right)=\phi\left(\lambda_{n}\right) \phi^{\prime}\left(\xi_{n}\right) .
$$

The hypothesis ensures $\Sigma\left|\lambda_{n}-\mu_{n}\right|<\infty$ and therefore $E(\lambda)=E(\mu)$ by [1].

In the context in which this result is used we generally have $\lambda_{n} \sim n$, whether $\phi$ is bounded or not, and the essential condition is

$$
\sum_{-\infty}^{\infty}\left|\phi(n) \phi^{\prime}(n)\right|<\infty .
$$

It is left for the reader to give regularity conditions under which this hypothesis ensures $E(\lambda)=E(\mu)$. For the particular application which we have in mind, the result given above is sufficient.

A familiar use of the counting function is to give the formula

$$
\log |F(x)|=\int_{-\infty}^{\infty} \frac{\Lambda(u)}{u} \frac{x}{x-u} d u
$$

where $\lambda_{k} \in R, \lambda_{k} \neq 0$, and

$$
F(x)=\lim _{R \rightarrow \infty} \prod_{\left|\lambda_{n}\right|<R}\left(1-\frac{x}{\lambda_{n}}\right) .
$$

The formula follows by partial integration if $\Lambda(u)=o\left(u^{2}\right)$, which is always the case in the applications intended here. 
Suppose now that $\Lambda(u)=[u-\phi(u)]$ where $\phi$ is continuous, $\phi^{\prime}<1$, and $0<\phi(0)$ $<1$. In a variety of investigations it is desirable to replace $\Lambda(u)$ in the above integral by the simpler expression $u-\phi(u)-\frac{1}{2}$. The error introduced by this substitution is

$$
E(x)=\int_{-\infty}^{\infty} \frac{\psi(u)}{u} \frac{x}{x-u} d u
$$

where $\psi(u)=[u-\phi(u)]-u+\phi(u)+\frac{1}{2}$.

Let us suppose that $|\phi|$ is bounded, $0<\phi(0)<1, \phi^{\prime}<1$, and $\Sigma_{-\infty}^{\infty}\left|\phi^{\prime \prime}\left(\xi_{n}\right)\right|<\infty$ whenever $\xi_{n} \in\left(\lambda_{n}, \lambda_{n+1}\right)$. Under these hypotheses we shall show that $|E(x)| \leqslant O(1)$ when $\inf _{n}\left|x-\lambda_{n}\right| \geqslant \varepsilon$, and $E(x) \leqslant O(1)$ when $x$ is unrestricted. Without loss of generality we confine attention to the case $x \rightarrow+\infty$; the case $x \rightarrow-\infty$ is similar. Then since $\int_{a}^{b} \psi(u) d u$ is bounded, as seen below,

$$
E(x)=\int_{x / 2}^{2 x} \frac{\psi(u)}{u} \frac{x}{x-u} d u+O(1), \quad x>0
$$

and it suffices to consider the latter integral.

To this end set $K(x, u)=x / u(x-u)$ and write

$$
\begin{aligned}
\int_{\lambda_{n}}^{\lambda_{n+1}} \psi(u) K(x, u) d u= & \int_{\lambda_{n}}^{\lambda_{n+1}} \psi(u)\left[K(x, u)-K\left(x, \lambda_{n}\right)\right] d u \\
& +K\left(x, \lambda_{n}\right) \int_{\lambda_{n}}^{\lambda_{n+1}} \psi(u) d u .
\end{aligned}
$$

Denoting the two expressions on the right by $I_{n}$ and $J_{n}$, respectively, we shall assess $I_{n}$ by use of the inequality

$$
\left|\frac{\partial K(x, u)}{\partial u}\right| \leqslant \frac{12}{(x-u)^{2}}, \quad \lambda_{n}<u<\lambda_{n+1} .
$$

This holds for $x / 2 \leqslant \lambda_{n} \leqslant \lambda_{n+1} \leqslant 2 x$ provided $x$ is not on the interval $\left[\lambda_{n}, \lambda_{n+1}\right]$. Since $\phi(u)=O(1)$ makes $\lambda_{n+1}-\lambda_{n}=O(1)$ we conclude that

$$
\left|I_{n}\right| \leqslant \int_{\lambda_{n}}^{\lambda_{n+1}} \frac{c}{(x-u)^{2}} d u
$$

where $c$ is constant. Let us form the sum of $I_{n}$ over all pairs $\left(\lambda_{n}, \lambda_{n+1}\right)$ on the interval $(1 / 2 x, 2 x)$ for which $\lambda_{n+1}<x-\varepsilon$, and also the sum over all pairs for which $\lambda_{n}>x+\varepsilon$. These sums are dominated by the integrals

$$
\int_{0}^{x-\varepsilon} \frac{c}{(x-u)^{2}} d u \text { and } \int_{x+\varepsilon}^{\infty} \frac{c}{(x-u)^{2}} d u
$$

respectively, each of which equals $c / \varepsilon$.

Let us now impose the condition inf $\left|x-\lambda_{n}\right| \geqslant \varepsilon$. In this case the pair $\left(\lambda_{n}, \lambda_{n+1}\right)$ for which $\lambda_{n}<x<\lambda_{n+1}$ is the only pair not included in the above calculation. Since $\lambda_{n+1}-\lambda_{n}=O(1)$, and since the integral is a principal value at $x$, the question whether $\Sigma I_{n}$ is bounded reduces effectively to the question whether

$$
\int_{x-\varepsilon}^{x+\varepsilon} \frac{\phi(x)-\phi(u)}{x-u} d u
$$

is bounded. It is readily verified that $\phi^{\prime}$ is bounded, however, and the result follows. 
Still assuming inf $\left|x-\lambda_{n}\right| \geqslant \varepsilon$, let us now turn to the second expression $J_{n}$. On the interval $\left(\lambda_{n}, \lambda_{n+1}\right)$ the function $\psi$ has the form $\psi(u)=c+\phi(u)-u$ where $c$ is a constant such that $\psi\left(\lambda_{n}\right)=1 / 2, \psi\left(\lambda_{n+1}\right)=-1 / 2$. This gives two expressions for $c$ which are consistent by virtue of the fact that $[u-\phi(u)]$ is the counting function for $\left\{\lambda_{n}\right\}$. Adding the two expressions, we get the symmetric form $2 c=\left(\lambda_{n+1}+\lambda_{n}\right)-$ $\left[\phi\left(\lambda_{n+1}\right)+\phi\left(\lambda_{n}\right)\right]$. On the other hand,

$$
\int_{\lambda_{n}}^{\lambda_{n+1}} \psi(u) d u=c\left(\lambda_{n+1}-\lambda_{n}\right)-\frac{1}{2}\left(\lambda_{n+1}^{2}-\lambda_{n}^{2}\right)+\int_{\lambda_{n}}^{\lambda_{n+1}} \phi(u) d u .
$$

The above expression for $c$ changes the right-hand side to

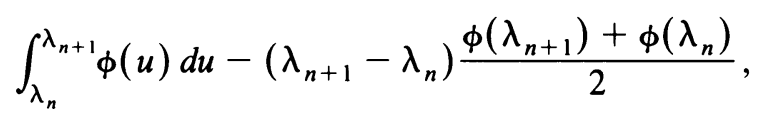

which is precisely the difference between the integral and its trapezoidal-rule approximation. Hence its magnitude does not exceed $C\left|\phi^{\prime \prime}\left(\xi_{n}\right)\right|$, where $\xi_{n} \in$ $\left(\lambda_{n}, \lambda_{n+1}\right)$ and where $C$ is a constant depending on $\sup \left(\lambda_{k+1}-\lambda_{k}\right)$. Since $\left|K\left(x, \lambda_{n}\right)\right| \leqslant 2 / \varepsilon$ for $\lambda_{n} \geqslant x / 2,\left|x-\lambda_{n}\right| \geqslant \varepsilon$, we see that the sum of the terms $J_{n}$ is bounded and the conclusion follows.

Suppose next that $\left|x-\lambda_{n}\right|<\varepsilon$ for some $n$. The number of such $\lambda$ is bounded by a constant, $N$, since $\phi(u)=O(1)$. Writing $\psi(u)$ as the sum of a smooth function and a jump function, we see that the contribution of these $\lambda_{k}$ to the integral produces a negative term plus a bounded term. The contribution of the other $\lambda_{k}$ is assessed as in the previous discussion, so that we get an upper bound. Actually the bound tends to $-\infty$ as $x \rightarrow \lambda_{n}$, in agreement with the fact that $F\left(\lambda_{n}\right)=0$.

5. Supplement to a theorem of Levinson. Let $N(u)$ be the number of $\lambda_{j}$ satisfying $\left|\lambda_{j}\right| \leqslant u$, where $\left\{\lambda_{j}\right\}$ is an arbitrary sequence of complex numbers. An important theorem of Levinson asserts that $\left\{e^{i \lambda_{n} x}\right\}$ is complete $L^{p}(-\pi, \pi)$ if

$$
\limsup _{r \rightarrow \infty}\left(\int_{1}^{r} \frac{N(t)}{t} d t-2 r+\frac{\log r}{q}\right)>-\infty .
$$

For a simple proof see [5, Theorem 8]. As a corollary Levinson deduces the completeness if $\left|\lambda_{n}\right| \leqslant|n|+/ 2 q,-\infty<n<\infty$, and also shows that the constant $1 / 2 q$ cannot be replaced by any larger constant.

Since (6) is unaffected if $N(t)$ is replaced by $N(t)+\phi(t)$, where $\phi(t) / t \in L(1, \infty)$, the completeness holds if

$$
\left|\lambda_{n}\right| \leqslant|n|+\frac{1}{2 q}+\phi(|n|)
$$

where $\phi$ is a sufficiently regular function satisfying $\Sigma_{1}^{\infty} \phi(n) / n<\infty$. Here we consider the case $\phi(n)=\beta / \log n, \beta \neq 0$, which just fails to satisfy this criterion. As seen by the following theorem, the question whether the set is complete depends on the value of $\beta$.

THEOREM 3. Let $\lambda_{1}$ be an arbitrary positive number and let $\lambda_{n}=n+1 / 2 q+$ $\beta / \log n$ for $n \geqslant 2$ where $\beta \geqslant 0$ and $1<p<\infty$. Then the set $\left\{1, e^{ \pm i \lambda_{n} x}\right\}$ is complete $L^{p}(-\pi, \pi)$ if $\beta \leqslant \min (1 / 4,1 / 2 q)$ and not if $\beta>\max (1 / 4,1 / 2 q)$. 
This result was stated without proof in [5] and was simultaneously communicated to one of us, again without proof, by D. R. Peterson.

Proof. It will be seen that the main difficulties are already overcome in $\S 4$, and that the rest of the proof requires little calculation. In fact, taking $\phi(u)=\alpha+$ $\beta / \log u$, we find that estimation of the canonical product associated with $\left\{0, \pm \lambda_{n}\right\}$ reduces effectively to the estimate

$$
\int_{2}^{\infty} \frac{2 a^{2}}{u\left(a^{2}-u^{2}\right)} \frac{d u}{\log u}=2 \log \log a+O(1), \quad a \rightarrow \infty .
$$

To establish (7) let us use the symbol $\doteq$ to denote equality within $O(1)$ as $a \rightarrow \infty$. Then

$$
\begin{aligned}
\int_{2}^{\infty} \frac{2 a^{2}}{u\left(a^{2}-u^{2}\right)} \frac{d u}{\log u} & \doteq \int_{2}^{\infty} \frac{2 a^{2}}{y\left(a^{2}+y^{2}\right)} \frac{\log y}{(\log y)^{2}+(\pi / 2)^{2}} d y \\
& \doteq \int_{2}^{\infty} \frac{2 a^{2}}{y\left(a^{2}+y^{2}\right)} \frac{d y}{\log y} \doteq \int_{2}^{a} \frac{2 a^{2}}{y\left(a^{2}+y^{2}\right)} \frac{d y}{\log y}
\end{aligned}
$$

Here the first $\doteq$ follows by integration over a contour in the first quadrant indented at 0 and $a$, and the others are more elementary. The last expression can be written

$$
\int_{2}^{a} \frac{2 d y}{y \log y}-\int_{2}^{a} \frac{2 y d y}{\left(a^{2}+y^{2}\right) \log y},
$$

which is $2 \log \log a+O(1)$ since the second integral does not exceed

$$
\frac{1}{\log 2} \int_{2}^{a} \frac{2 y d y}{a^{2}}
$$

Suppose now that $\lambda_{n}=n+\alpha+\beta / \log n$ for $n \geqslant 1$, where $\alpha$ and $\beta$ are constant and where $\log 1$ is replaced by any suitable value to give $\lambda_{1}$. If $F(x)$ denotes the canonical product associated with $\left\{ \pm \lambda_{n}\right\}$ we can replace $F$, in the study of completeness, by another canonical product $G$ with zeros $\pm \mu_{n}$. The counting function for $\left\{\mu_{n}\right\}$ is $\Lambda(u)=[u-\alpha-\beta / \log u], u \gg 1$. When only an upper bound is needed, or when $\inf _{k}\left|x-\mu_{k}\right| \geqslant \varepsilon>0$, the function $G$ in turn can be replaced by $H$ where

$$
\log H(x)=\int_{2}^{\infty}\left(u-\alpha-\frac{\beta}{\log u}-\frac{1}{2}\right) \frac{2 x^{2}}{u\left(x^{2}-u^{2}\right)} d u .
$$

(All of these assertions follow by obvious choice of $\phi$ in $\$ 4$.)

The only troublesome term in the evaluation of $H$ is given by (7) with $a=x$, and hence

$$
\log H(x)=-\left(\alpha+\frac{1}{2}\right)(2 \log x)-2 \beta \log \log x+O(1)
$$

as $x \rightarrow \infty$. Adding the factor $x$ corresponding to $\lambda_{0}=0$ we see that the relevant estimate for Theorem 3 is

$$
C_{1} x^{-2 \alpha}(\log x)^{-2 \beta} \leqslant x H(x) \leqslant C_{2} x^{-2 \alpha}(\log x)^{-2 \beta}
$$

which holds for large $x$ with positive constants $C_{i}$. 
Suppose, now, that $\alpha=1 / 2 q$ and $\beta>1 / 2 q$. In this case $x H \in L^{q}(-\infty, \infty)$ and, since $\log |F| \leqslant \log |H|+O(1)$, the same is true of $x F$. Hence $x F$ admits the representation

$$
x F(x)=\int_{-\pi}^{\pi} e^{i x t} f(t) d t, \quad f \in L^{p}(-\pi, \pi),
$$

if $q \leqslant 2$ and it follows that $\left\{1, e^{ \pm i \lambda_{n} x}\right\}$ is not complete.

In like manner, if $\alpha=1 / 2 q$ and $\beta \leqslant 1 / 2 q$, we see not only that $x H$ fails to be in $L^{q}$, but even that $[x H]^{q} \geqslant 1 /(x \log x)$ for large $x$. A similar estimate holds for $|x F|$ in the set where $\left|x-\lambda_{n}\right|>\frac{1}{4}$, say, and shows that $x F$ is not in $L^{q}$. It follows that the set $\left\{1, e^{ \pm i \lambda_{n} x}\right\}$ is complete if $p \leqslant 2$. Otherwise there would be a representation

$$
x F(x) K(x)=\int_{-\pi}^{\pi} e^{i x t} f(t) d t, \quad f \in L^{p}(-\pi, \pi),
$$

with $K$ of type 0 . But in fact $K$ must be a polynomial, since $|E(\lambda)|<\infty$; and even if the degree of $K$ is 0 , we would have $x F \in L^{q}$. This completes the proof.

When Levinson's criterion (6) is applied to $\left\{0, \pm \lambda_{n}\right\}$ with $\Lambda(u)$ as above, the correct choice for $N$ is $N(t)=1+2 \Lambda(t)$. The criterion for completeness is then

$$
\limsup _{r \rightarrow \infty}\left(\int_{1}^{r} \frac{\Lambda(t)}{t} d t-r+\frac{1+q}{2 q} \log r\right)>-\infty .
$$

In the context of Theorem 3 we have, effectively, $\Lambda(u)=u-\alpha-\beta / \log u-\frac{1}{2}$ and the expression following the lim sup is $(1 / 2 q-\alpha) \log r-\beta \log \log r$. The criterion is fulfilled when $\alpha<1 / 2 q$, also when $\alpha=1 / 2 q$ and $\beta=0$, but not when $\alpha=1 / 2 q$ and $\beta>0$. To one familiar with the proof of (6), the fact that such a regular distribution of $\left\{\lambda_{n}\right\}$ can lead to completeness without satisfying (6) is surprising. Another surprising aspect of Theorem 3 was already mentioned in [5]; namely, Theorem 3 shows that $\left|\lambda_{n}-\mu_{n}\right|=o(1)$ is not enough to give $E(\lambda)=E(\mu)$ even if this condition is strengthened to $\left|\lambda_{n}-\mu_{n}\right|<\varepsilon / \log n$. Here again, the behavior does not hinge upon irregularity of $\left\{\lambda_{n}\right\}$ or $\left\{\mu_{n}\right\}$.

\section{A canonical product. Let}

$$
\lambda_{n}= \begin{cases}n+\varepsilon, & n>0 \\ 0, & n=0 \\ n-\varepsilon, & n<0\end{cases}
$$

where $\varepsilon$ is positive, and let $F(z)$ be the canonical product with these zeros:

$$
F(z)=z \prod_{n=1}^{\infty}\left(1-z^{2} / \lambda_{n}^{2}\right)
$$

For the results that follow, we shall require both an integral repesentation for $F(z)$ and a simple formula for $F^{\prime}\left(\lambda_{n}\right)$.

The following lemma is essentially contained in [3, p. 67]. 
LEMMA 1. If $0<\varepsilon<\frac{1}{4}$ and $F(z)$ is given by (8), then

$$
F(z)=c \int_{-\pi}^{\pi} f(t) e^{i z t} d t
$$

where

$$
f(t)=\left(\cos \frac{1}{2} t\right)^{2 \varepsilon-1} \sin \frac{1}{2} t .
$$

Proof. Write $G(z)=\int_{-\pi}^{\pi} f(t) e^{i z t} d t$. Since $\varepsilon$ is positive, $f$ is integrable over the interval $(-\pi, \pi)$ and hence $G(z)$ is an entire function of exponential type at most $\pi$. As pointed out by Levinson [3, p. 11] $G\left(\lambda_{n}\right)=0$ for every $n$.

ASSERTION. $G(z)$ can vanish only at the $\lambda_{n}$. Suppose to the contrary that $G(z)$ were zero for some other value of $z$, say $z=\mu$. Then integration by parts shows that

$$
\frac{G(z)}{z-\mu}=\int_{-\pi}^{\pi} g(t) e^{i z t} d t
$$

with

$$
g(t)=-i e^{-i \mu t} \int_{-\pi}^{t} f(x) e^{i \mu x} d x .
$$

Accordingly, $g$ is a nontrivial function in $L^{2}(-\pi, \pi)$ orthogonal to $e^{i \lambda_{n} t}$ for every $n$. But this is absurd since the condition $\left|\lambda_{n}-n\right| \leqslant \frac{1}{4}$ guarantees that the system $\left\{e^{i \lambda_{n} t}\right\}$ is complete $L^{2}(-\pi, \pi)$ [3, Theorem 4]. The contradiction proves the assertion.

By Hadamard's factorization theorem, we can write $G(z)=A e^{B z} F(z)$. Since $F(z)$ and $G(z)$ are both odd, $B=0$ and hence $G(z)=A F(z)$. This proves the lemma.

REMARK. The proof of the lemma reveals that the system $\left\{e^{i \lambda_{n} t}\right\}$ is not only complete $L^{2}(-\pi, \pi)$ but also exact. Indeed,

$$
\frac{G(z)}{z-\lambda_{n}}=\int_{-\pi}^{\pi} g_{n}(t) e^{i z t} d t
$$

where

$$
g_{n}(t)=-i e^{-i \lambda_{n} t} \int_{-\pi}^{t} f(x) e^{i \lambda_{n} x} d x
$$

Thus, $g_{n}$ is a nontrivial function in $L^{2}(-\pi, \pi)$ orthogonal to $e^{i \lambda_{k} t}$ whenever $k \neq n$, and the system $\left\{e^{i \lambda_{k} t}\right\}_{k \neq n}$ is incomplete $L^{2}(-\pi, \pi)$.

LEMMA 2. If $\lambda_{n}=n+\varepsilon(n=1,2,3, \ldots)$, where $\varepsilon>-1$, and

$$
H(z)=\prod_{n=1}^{\infty}\left(1-\frac{z^{2}}{\lambda_{n}^{2}}\right),
$$

then

$$
H^{\prime}\left(\lambda_{n}\right)=(-1)^{n} \Gamma^{2}(1+\varepsilon) \frac{\Gamma(n)}{\Gamma(n+1+2 \varepsilon)} .
$$

Proof. Put $\mu=\lambda_{1}=1+\varepsilon$. We claim that

$$
H(z)=\frac{\Gamma^{2}(\mu)}{\Gamma(\mu+z) \Gamma(\mu-z)} .
$$


Call the right side $G(z)$. Then $G(z)$ is entire of order 1 and has zeros $\pm \lambda_{n}$ $(n=1,2,3, \ldots)$. By Hadamard's theorem, $G(z)=e^{a z} H(z)$. But $G(z)$ and $H(z)$ are both even, and hence $a=0$. Thus $G(z)=H(z)$.

Thus,

$$
H^{\prime}(z)=\Gamma^{2}(\mu)\left\{\frac{1}{\Gamma(\mu+z)} \frac{\Gamma^{\prime}(\mu-z)}{\Gamma^{2}(\mu-z)}-\frac{1}{\Gamma(\mu-z)} \frac{\Gamma^{\prime}(\mu+z)}{\Gamma^{2}(\mu+z)}\right\}
$$

and

$$
\begin{aligned}
H^{\prime}\left(\lambda_{n}\right) & =\frac{\Gamma^{2}(\mu)}{\Gamma\left(\mu+\lambda_{n}\right)} \cdot \lim _{z \rightarrow \lambda_{n}} \frac{\Gamma^{\prime}(\mu-z)}{\Gamma^{2}(\mu-z)} \\
& =\frac{\Gamma^{2}(\mu)}{\Gamma(n+1+2 \varepsilon)} \cdot \lim _{z \rightarrow-n+1} \frac{\Gamma^{\prime}(z)}{\Gamma^{2}(z)} .
\end{aligned}
$$

We complete the proof by showing that

$$
\lim _{z \rightarrow-n} \frac{\Gamma^{\prime}(z)}{\Gamma^{2}(z)}=(-1)^{n+1} n ! \quad(n=0,1,2, \ldots) .
$$

Suppose first that $n=0$ and write

$$
\begin{aligned}
\frac{\Gamma^{\prime}(z)}{\Gamma^{2}(z)} & =\frac{1}{\Gamma(z)} \frac{\Gamma^{\prime}(z)}{\Gamma(z)}=\frac{\psi(z)}{\Gamma(z)} \\
& =\frac{1}{\Gamma(z)}\left\{-\frac{1}{z}-\gamma+\sum_{n=1}^{\infty} \frac{z}{n(n+z)}\right\}
\end{aligned}
$$

Since $1 / z \Gamma(z) \rightarrow 1$ as $z \rightarrow 0$, the result for $n=0$ follows. Proceed by induction. Since $\Gamma(z+1)=z \Gamma(z)$ and $\psi(z+1)=\psi(z)+1 / z$, we find

$$
\begin{aligned}
\lim _{z \rightarrow-(n+1)} \frac{\Gamma^{\prime}(z)}{\Gamma^{2}(z)} & =\lim _{z \rightarrow-n} \frac{\psi(z-1)}{\Gamma(z-1)}=\lim _{z \rightarrow-n} \frac{\psi(z)-1 /(z-1)}{\Gamma(z) /(z-1)} \\
& =\lim _{z \rightarrow-n}\left(\frac{\psi(z)}{\Gamma(z)}(z-1)-\frac{1}{\Gamma(z)}\right) \\
& =(-1)^{n+1} n !(-n-1)=(-1)^{n+2}(n+1) !
\end{aligned}
$$

This completes the proof.

7. On Kadec's “ $\frac{1}{4}$-theorem". Kadec's “ $\frac{1}{4}$-theorem" asserts that the system of exponentials $\left\{e^{i \lambda_{n} t}\right\}$ forms a Riesz basis for $L^{2}(-\pi, \pi)$ whenever each $\lambda_{n}$ is real and $\left|\lambda_{n}-n\right| \leqslant L<\frac{1}{4}(-\infty<n<\infty)$. In this section we shall investigate some of the ways in which Kadec' result is the best possible.

It is not difficult to see that the condition

$$
\left|\lambda_{n}-n\right|<\frac{1}{4} \quad(-\infty<n<\infty)
$$

is not strong enough to guarantee that the system $\left\{e^{i \lambda_{n} t}\right\}$ is a Riesz basis for $L^{2}(-\pi, \pi)$ for the trivial reason that it permits the system to have an excess. Indeed, if we take $\mu_{n}=n-\frac{1}{4}, \mu_{-n}=-\mu_{n}(n=1,2,3, \ldots)$, and then choose $\left\{\lambda_{n}\right\}$ so that (9) 
holds and $\Sigma\left|\lambda_{n}-\mu_{n}\right|<\infty$, then $\left\{e^{i \mu_{n} t}\right\}_{n \neq 0}$ is complete $L^{2}(-\pi, \pi)$ (see, e.g., [7, p. 122]) and, hence, so is $\left\{e^{i \lambda_{n} t}\right\}_{n \neq 0}[1$, p. 66]. Thus, (9) cannot even guarantee that the system $\left\{e^{i \lambda_{n} t}\right\}$ is exact.

We are going to show that condition (9) is not strong enough to ensure that $\left\{e^{i \lambda_{n} t}\right\}$ is a Riesz basis for $L^{2}(-\pi, \pi)$ even if it is exact. For this purpose we shall make use of the Paley-Wiener space $P$ consisting of all entire functions of exponential type at most $\pi$ that are square integrable on the real axis. The inner product of two functions $F$ and $G$ in $P$ is, by definition,

$$
(F, G)=\int_{-\infty}^{\infty} F(x) \overline{G(x)} d x .
$$

By virtue of the Paley-Wiener theorem, the complex Fourier transform

$$
f(t) \rightarrow \frac{1}{2 \pi} \int_{-\pi}^{\pi} f(t) e^{-i z t} d t
$$

is an isometric isomorphism from $L^{2}(-\pi, \pi)$ onto all of $P$. When the $\lambda_{n}$ are real, the exponentials $e^{i \lambda_{n} t}$ are sent to the "reproducing" functions

$$
K_{n}(z)=\frac{\sin \pi\left(z-\lambda_{n}\right)}{\pi\left(z-\lambda_{n}\right)}
$$

so that $\left(F, K_{n}\right)=F\left(\lambda_{n}\right)$ for every $F \in P$.

By means of the Fourier isometry, problems involving complex exponentials in $L^{2}(-\pi, \pi)$ can be examined via their transform image in $P$. Thus, for example, the system $\left\{e^{i \lambda_{n} t}\right\}$ is a Riesz basis for $L^{2}(-\pi, \pi)$ if and only if the interpolation problem $F\left(\lambda_{n}\right)=c_{n}$ is uniquely solvable for $F$ in $P$ whenever $\left\{c_{n}\right\} \in l^{2}$, and for these sequences only (see, e.g., [7, p. 170]).

THEOREM 4. If

$$
\lambda_{n}= \begin{cases}n+\frac{1}{4}, & n>0, \\ 0, & n=0, \\ n-\frac{1}{4}, & n<0\end{cases}
$$

then the system $\left\{e^{i \lambda_{n} t}\right\}$ is not a Riesz basis for $L^{2}(-\pi, \pi)$.

Proof. Suppose it were. Then the system of reproducing functions $\left\{K_{n}(z)\right\}$ would be a Riesz basis for $P$, since the Fourier transform is an isometry. Put

$$
F_{n}(z)=\frac{F(z)}{F^{\prime}\left(\lambda_{n}\right)\left(z-\lambda_{n}\right)},
$$

where $F(z)=z \Pi_{1}^{\infty}\left(1-z^{2} / \lambda_{n}^{2}\right)$. Then $F_{n}\left(\lambda_{k}\right)=\delta_{n k}$, and the Remark following Lemma 1 shows that each $F_{n}$ belongs to $P$. Accordingly, $\left\{F_{n}(z)\right\}$ is "biorthogonal" to $\left\{K_{n}(z)\right\}$ in $P$ (see $\S 8$ for the definition) and so must also be a Riesz basis for $P$ [7]. In particular, the series

$$
\sum_{-\infty}^{\infty} c_{n} \frac{F(z)}{F^{\prime}\left(\lambda_{n}\right)\left(z-\lambda_{n}\right)}
$$


must converge in the topology of $P$, and hence pointwise, whenever $\left\{c_{n}\right\} \in L^{2}$. By the converse to Hölder's inequality, this can happen only if

$$
\sum_{n \neq 0}\left|\frac{1}{\lambda_{n} F^{\prime}\left(\lambda_{n}\right)}\right|^{2}<\infty
$$

But by Lemma 2,

$$
F^{\prime}\left(\lambda_{n}\right)=\lambda_{n}(-1)^{n} \Gamma^{2}\left(\frac{5}{4}\right) \frac{\Gamma(n)}{\Gamma(n+3 / 2)} \quad(n=1,2,3, \ldots),
$$

and Stirling's formula, $\Gamma(x+1) \sim(2 \pi)^{1 / 2} x^{x+1 / 2} e^{-x}$, shows that $\left|F^{\prime}\left(\lambda_{n}\right)\right| \sim c n^{-1 / 2}$ as $n \rightarrow \infty$. Thus, $\sum_{n \neq 0}\left|1 / \lambda_{n} F^{\prime}\left(\lambda_{n}\right)\right|^{2}=\infty$, and the contradiction proves the theorem.

COROllaRY. The condition $\left|\lambda_{n}-n\right|<\frac{1}{4}(-\infty<n<\infty)$ is not sufficient to ensure that the system $\left\{e^{i \lambda_{n} t}\right\}$ is a Riesz basis for $L^{2}(-\pi, \pi)$, even if it is exact.

Proof. Let $\mu_{n}=n+\frac{1}{4}, \mu_{-n}=-\mu_{n}(n=1,2,3, \ldots)$, and $\mu_{0}=0$, and choose $\left\{\lambda_{n}\right\}$ so that $\left|\lambda_{n}-n\right|<\frac{1}{4}$ and $\Sigma\left|\lambda_{n}-\mu_{n}\right|<\infty$. Since $\left\{e^{i \mu_{n} t}\right\}$ is exact (see the Remark following Lemma 1) so is $\left\{e^{i \lambda_{n} t}\right\}\left[1\right.$, p. 66]. The claim is that $\left\{e^{i \lambda_{n} t}\right\}$ is not a Riesz basis for $L^{2}(-\pi, \pi)$. Suppose it were. Then there is a constant $\varepsilon>0$ such that $\left\{e^{i \gamma_{n} t}\right\}$ is also a Riesz basis for $L^{2}(-\pi, \pi)$ provided $\left|\lambda_{n}-\gamma_{n}\right|<\varepsilon$ for all $n$ [7, p. 191]. Since $\left|\lambda_{n}-\mu_{n}\right| \rightarrow 0$, we may choose $N$ so large that $\left|\lambda_{n}-\mu_{n}\right|<\varepsilon$ when $|n|>N$, and conclude that the system $\left\{e^{i \lambda_{n} t}\right\}_{|n| \leqslant N} \cup\left\{e^{i \mu_{n} t}\right\}_{|n|>N}$ is a Riesz basis for $L^{2}(-\pi, \pi)$. But it is a simple matter to show that the basis properties of a system of complex exponentials is unaffected if one of the exponents is replaced by another number. Replacing the $\lambda_{n}$ 's $(|n| \leqslant N)$ by the corresponding $\mu_{n}$ 's, we conclude that the system $\left\{e^{i \mu_{n} t}\right\}$ is a Riesz basis for $L^{2}(-\pi, \pi)$. But this contradicts Theorem 4 and the proof is complete.

8. The system biorthogonal to $\left\{1, e^{ \pm i(n+1 / 4) t}\right\}$. Fundamental to the study of bases in a separable Hilbert space $H$ is the notion of a biorthogonal system (see, e.g., [6 and 7] and the references therein). Two sequences $\left\{f_{n}\right\}$ and $\left\{g_{n}\right\}$ of elements from $H$ are said to be biorthogonal if $\left(f_{n}, g_{m}\right)=\delta_{n m}$.

It is easy to show that a sequence $\left\{f_{n}\right\}$ possesses a biorthogonal sequence if and only if it is minimal, i.e., if none of its elements can be approximated by linear combinations of the others. If this is the case, then a biorthogonal sequence will be uniquely determined if and only if $\left\{f_{n}\right\}$ is exact.

In this section we shall investigate further the system biorthogonal to $\left\{1, e^{ \pm i(n+1 / 4) t}\right\}$. Put

$$
G(z)=\int_{-\pi}^{\pi} f(t) e^{i z t} d t
$$

where $f(t)=\left(\cos \frac{1}{2} t\right)^{-1 / 2} \sin \frac{1}{2} t$. Let $\lambda_{n}=n+\frac{1}{4}, \lambda_{-n}=-\lambda_{n}(n=1,2,3, \ldots)$, and $\lambda_{0}=0$. It follows at once from Lemma 1 and the Remark following it that the system $\left\{e^{i \lambda_{n} t}\right\}$ is exact in $L^{2}(-\pi, \pi)$ and that its (unique) biorthogonal system is 
given by $\left\{g_{n} / G^{\prime}\left(\lambda_{n}\right)\right\}$, where

$$
g_{n}(t)=-i e^{-i \lambda_{n} t} \int_{-\pi}^{t} f(x) e^{i \lambda_{n} x} d x
$$

THEOREM 5. If

$$
\lambda_{n}= \begin{cases}n+\frac{1}{4}, & n>0, \\ 0, & n=0, \\ n-\frac{1}{4}, & n<0,\end{cases}
$$

then the system $\left\{f_{n}\right\}$ biorthogonal to $\left\{e^{i \lambda_{n} t}\right\}$ in $L^{2}(-\pi, \pi)$ is bounded, i.e., $\sup _{n}\left\|f_{n}\right\|<$ $\infty$.

Proof. We have $\left\|f_{n}\right\|=\left\|g_{n}\right\| /\left|G^{\prime}\left(\lambda_{n}\right)\right|$, where $G$ and $g_{n}$ are given by (10) and (11), respectively. We begin by showing that

$$
\left\|g_{n}\right\|=\left\|\int_{-\pi}^{t} f(x) e^{i \lambda_{n} x} d x\right\|=O\left(\frac{1}{\sqrt{\lambda_{n}}}\right) \quad \text { as } n \rightarrow \infty .
$$

Since $f(x)$ is odd, a similar assertion is true for $n \rightarrow-\infty$. Put $I_{\lambda}(t)=\int_{-\pi}^{t} f(x) e^{i \lambda x} d x$ for $\lambda>0$ and $-\pi \leqslant t \leqslant \pi$. We define

$$
g(t)=f(t)-(\sqrt{2 / \pi-|t|}-\sqrt{2 / \pi}) \operatorname{sgn} t
$$

for $-\pi<t<\pi$, and $g( \pm \pi)=0$. Let

$$
J_{\lambda}(t)=\int_{-\pi}^{t} g(x) e^{i \lambda x} d x
$$

Then

$$
\left\|I_{\lambda}\right\| \leqslant\left\|J_{\lambda}\right\|+\left\|\int_{-\pi}^{t}(f(x)-g(x)) e^{i \lambda x} d x\right\| .
$$

It is easy to see that $g(t)$ is of class $C^{1}$ on $[-\pi, \pi]$, and integration by parts yields

$$
J_{\lambda}(t)=\frac{1}{i \lambda}\left(g(t) e^{i \lambda t}-\int_{-\pi}^{t} g^{\prime}(x) e^{i \lambda x} d x\right) .
$$

Accordingly,

$$
\left\|J_{\lambda}\right\|=O(1 / \lambda) \text { as } \lambda \rightarrow \infty \text {. }
$$

Put

$$
H_{\lambda}(t)=\int_{-\pi}^{t} \frac{1}{\sqrt{\pi-|x|}} e^{i \lambda x} d x
$$

Then

$$
\left\|H_{\lambda}\right\|^{2}=\int_{-\pi}^{\pi}\left|H_{\lambda}(t)\right|^{2} d t=\left(\int_{-\pi}^{0}+\int_{0}^{\pi}\right)\left|H_{\lambda}(t)\right|^{2} d t .
$$

When $-\pi \leqslant t \leqslant 0$,

$$
H_{\lambda}(t)=\int_{-\pi}^{t} \frac{1}{\sqrt{\pi+x}} e^{i \lambda x} d x=\frac{e^{-i \pi \lambda}}{\sqrt{\lambda}} \int_{0}^{(\pi+t) \lambda} \frac{e^{i u}}{\sqrt{u}} d u
$$


(put $u=(\pi+x) \lambda)$. Thus

$$
\int_{-\pi}^{0}\left|H_{\lambda}(t)\right|^{2} d t=\frac{1}{\lambda} \int_{-\pi}^{0}|G((\pi+t) \lambda)|^{2} d t,
$$

where we have put $G(t)=\int_{0}^{t}\left(e^{i u} / \sqrt{u}\right) d u$. As $\lambda \rightarrow \infty$,

$$
\int_{-\pi}^{0}|G((\pi+t) \lambda)|^{2} d t \rightarrow \pi|G(\infty)|^{2} \neq 0 .
$$

The integral $\int_{0}^{\pi}\left|H_{\lambda}(t)\right|^{2} d t$ is treated similarly, and we obtain

$$
\left\|H_{\lambda}\right\|=O(1 / \sqrt{\lambda}) \text { as } \lambda \rightarrow \infty .
$$

Direct integration shows that

$$
\left\|\int_{-\pi}^{t} \sqrt{\frac{2}{\pi}} e^{i \lambda x} d x\right\|=O\left(\frac{1}{\lambda}\right) \quad \text { as } \lambda \rightarrow \infty .
$$

Combining (13)-(16), we find that $\left\|I_{\lambda}\right\|=O(1 / \sqrt{\lambda})$ as $\lambda \rightarrow \infty$. This establishes (12).

To complete the proof, we show that for some positive constant $A$,

$$
\left|G^{\prime}\left(\lambda_{n}\right)\right| \sim A / \sqrt{\lambda_{n}} \text { as } n \rightarrow \infty \text {. }
$$

But by Lemma $1, G(z)=c z \prod_{n=1}^{\infty}\left(1-z^{2} / \lambda_{n}^{2}\right)$, and so, by Lemma 2 ,

$$
G^{\prime}\left(\lambda_{n}\right)=c \lambda_{n}(-1)^{n} \Gamma^{2}\left(\frac{5}{4}\right) \frac{\Gamma(n)}{\Gamma(n+3 / 2)}
$$

for $n=1,2,3, \ldots$ Applying Stirling's formula, we obtain (17). The proof is complete since (12) and (17) show that $\sup _{n}\left\|f_{n}\right\|<\infty$.

REMARKS. Theorem 4 and the remarks preceding it show that the interpolation problem

$$
F\left(\lambda_{n}\right)=c_{n} \quad(-\infty<n<\infty),
$$

where $F$ belongs to the Paley-Wiener space $P$, cannot be solved for every squaresummable sequence $\left\{c_{n}\right\}$. On the other hand, the functions

$$
G(z) / G^{\prime}\left(\lambda_{n}\right)\left(z-\lambda_{n}\right),
$$

being the Fourier transforms of the $f_{n}$ 's, must belong to $P$ and, according to Theorem 5 , must also be uniformly bounded in norm. Thus the series

$$
F(z)=\sum_{-\infty}^{\infty} c_{n} \frac{G(z)}{G^{\prime}\left(\lambda_{n}\right)\left(z-\lambda_{n}\right)}
$$

converges absolutely whenever $\left\{c_{n}\right\}$ is summable, and so represents the unique solution to (18). Put another way, the Lagrange expansion

$$
F(z)=\sum_{-\infty}^{\infty} F\left(\lambda_{n}\right) \frac{G(z)}{G^{\prime}\left(\lambda_{n}\right)\left(z-\lambda_{n}\right)}
$$

is valid for any function $F$ in $P$ for which $\Sigma\left|F\left(\lambda_{n}\right)\right|<\infty$. Since the system of exponentials $\left\{e^{i \lambda_{n} t}\right\}$ is complete $L^{2}(-\pi, \pi)$, so is its biorthogonal system $\left\{f_{n}\right\}$ [8], and it follows that the functions $\left\{G(z) / G^{\prime}\left(\lambda_{n}\right)\left(z-\lambda_{n}\right)\right\}$ are necessarily complete in $P$. 
Thus, the expansion above is valid on a dense subspace of $P$. If it could be shown that this expansion is valid for every function belonging to $P$, then we could conclude first that the system $\left\{G(z) / G^{\prime}\left(\lambda_{n}\right)\left(z-\lambda_{n}\right)\right\}$ is a basis for $P$, next that $\left\{f_{n}\right\}$ is a basis for $L^{2}(-\pi, \pi)$, and finally that its biorthogonal system $\left\{e^{i \lambda_{n} t}\right\}$ is also a basis for $L^{2}(-\pi, \pi)$. At present, there is no known example of a basis of complex exponentials that is not a Riesz basis.

9. Functions of sine type. One of the most natural ways of generating Riesz bases of complete exponentials $\left\{e^{i \lambda_{n} t}\right\}$ is by means of an entire generating function which vanishes at the $\lambda_{n}$ 's and, in some sense, resembles $\sin \pi z$.

Definition. An entire function $F(z)$ of exponential type $\pi$ is said to be of sine type if

(1) its zeros $\lambda_{n}$ are separated, i.e., $\inf _{n \neq m}\left|\lambda_{n}-\lambda_{m}\right|>0$, and

(2) there exist positive constants $A, B$, and $H$ such that

$$
A e^{\pi|y|} \leqslant|F(x+i y)| \leqslant B e^{\pi|y|}
$$

whenever $x$ and $y$ are real and $|y| \geqslant H$.

The following theorem is fundamental (see, e.g., [7, p. 172]).

THEOREM 6 (LEVIN-Goluvin). If $\left\{\lambda_{n}\right\}$ is the set of zeros of a function of sine type, then the system $\left\{e^{i \lambda_{n} t}\right\}$ forms a Riesz basis for $L^{2}(-\pi, \pi)$.

The following theorem exhibits a large class of Riesz bases of complex exponentials that cannot be obtained in this way.

THEOREM 7. If $0<\varepsilon<\frac{1}{4}$ and

$$
\lambda_{n}= \begin{cases}n+\varepsilon, & n>0 \\ 0, & n=0 \\ n-\varepsilon, & n<0\end{cases}
$$

then the system $\left\{e^{i \lambda_{n} t}\right\}$ is a Riesz basis for $L^{2}(-\pi, \pi)$ but $\left\{\lambda_{n}\right\}$ is not the set of zeros of a function of sine type.

Proof. The first assertion follows at once from Kadec's “ $\frac{1}{4}$-theorem". For the second, we argue by contradiction. Suppose then that there were a function $F(z)$ of sine type with zero set $\left\{\lambda_{n}\right\}$. Then $\inf _{n}\left|F^{\prime}\left(\lambda_{n}\right)\right|>0$ (see, e.g., [7, p. 173]). By Hadamard's theorem, we can write

$$
F(z)=A z e^{B z} \prod_{n=1}^{\infty}\left(1-z^{2} / \lambda_{n}^{2}\right) .
$$

If $\operatorname{Re} B \leqslant 0$, then by Lemma 2 together with Stirling's formula,

$$
\left|F^{\prime}\left(\lambda_{n}\right)\right| \sim c e^{n \operatorname{Re} B} n^{-2 \varepsilon} \quad \text { as } n \rightarrow \infty,
$$

and hence $\left|F^{\prime}\left(\lambda_{n}\right)\right| \rightarrow 0$ as $n \rightarrow \infty$. If $\operatorname{Re} B>0$, then we conclude in similar fashion that $\left|F^{\prime}\left(\lambda_{n}\right)\right| \rightarrow 0$ as $n \rightarrow-\infty$. The contradiction proves the theorem. 


\section{REFERENCES}

1. W. O. Alexander, Jr. and R. M. Redheffer, The excess of sets of complex exponentials, Duke Math. J. 34 (1967), 59-72.

2. G. H. Hardy, J. E. Littlewood and G. Pólya, Inequalities, 2nd ed., Cambridge Univ. Press, London, 1952.

3. N. Levinson, Gap and density theorems, Amer. Math. Soc. Colloq. Publ., vol. 26, Amer. Math. Soc., Providence, R. I., 1940.

4. R. M. Redheffer, Ganze Funktionen und Vollständigkeit, Osterreich Akad. Wiss. Math.-Natur. K1. Denkschr. 6 (1957), 96-99.

5. __ Completeness of sets of complex exponentials, Adv. in Math. 24 (1977), 1-62.

6. I. Singer, Bases in Banach spaces. I, Springer-Verlag, Berlin and New York, 1970.

7. R. M. Young, An introduction to nonharmonic Fourier series, Academic Press, New York, 1980.

8. On complete biorthogonal systems, Proc. Amer. Math. Soc. 83 (1981), 537-540.

9. A. Zygmund, Trigonometric series, 2nd ed., Cambridge Univ. Press, London, 1959.

Department of Mathematics, University of California, Los Angeles, California 90024

Department of Mathematics, Oberlin College, Oberlin, Ohio 44074 